\title{
Growth and Dry Matter Partitioning Efficiency of Blackgram Genotypes Influenced by Leaf Curl Disease under Natural Field Conditions
}

\author{
V. Dinesh Rahul ${ }^{1 *}$, G. Rama Rao ${ }^{2}$, K. Jayalalitha ${ }^{1}$, \\ M. Adinarayana ${ }^{3}$ and V. Srinivasa $\operatorname{Rao}^{4}$ \\ ${ }^{1}$ Department of Crop Physiology, ${ }^{4}$ Department Agricultural Statistics, \\ Agricultural College, Bapatla, Acharya N.G. Ranga Agricultural University, \\ Bapatla - 522 101, Andhra Pradesh, India \\ ${ }^{2} R A R S$, Chintapalle, India \\ ${ }^{3} R R U$, Plant pathology, RARS, Lam, India \\ *Corresponding author
}

\section{A B S T R A C T}

\section{Ke yw o r d s \\ Blackgram, Thrips population, Leaf curl disease, GBNV, Weather, Temperature, Relative Humidity \\ Article Info \\ Accepted: \\ 12 August 2020 \\ Available Online: \\ 10 September 2020}

A field experiment was conducted to evaluate the performance of twenty five blackgram genotypes under naturally epidemic conditions to leaf curl disease. The experiment was under taken during the late rabi 2018-19 and 2019-20 in a randomized block design replicated thrice. The investigation has focused on the study of variation in the dry matter production efficiency of the genotypes as influenced by leaf curl disease infection. The results obtained indicated that the leaf curl disease has severely affected the growth and dry matter partitioning efficiency of all the blackgram genotypes tested. The genotypes that show resistance to leaf curl disease showed less reduction dry weights. Leaf dry weight, pod dry weight and total dry matter showed a highly significant negative correlation with leaf curl disease at 60 DAS and maturity. The resistant genotype TU 40 showed higher partitioning efficiency and pod dry weights.

\section{Introduction}

India is the largest producer and consumer of pulses in the world (Chauhan et al., 2016). Blackgram is one of the most important pulse crop grown in India. It is also the third most cultivated pulse crop in terms of area after pigeonpea and chickpea with a production of 35.6 lakh tones during 2017-18 (ASG, 2018). Blackgram is also a nutritionally important crop with a good amount of dietary protein content.

India is contributing $70 \%$ of the world's blackgram production yet India imports huge quantities majorly from Myanmar. This can be attributed to the lower genetic yield potential of blackgram, lesser partitioning efficiency and lower concern given to the crop grown on marginal lands with higher 
biotic and abiotic stresses. The major biotic stress factors that influence the crop growth of blackgram are a class of viral diseases which include mungbean yellow mosaic virus (MYMV), leaf curl virus disease caused by groundnut bud necrosis virus (GBNV) and urdbean leaf crinkle virus (ULCV) (Biswas et al., 2009).

It is clear from various reports that virus infection reduced the plant growth and dry biomass and fresh weights of tomato plants infected with yellow mosaic or aucuba. The virus infected plants showed reduced carbohydrate and residual dry matter contents in their stems (Caldwell, 1934). In the present study the genotype screening was carried out based on the retention of their partitioning efficiency among the blackgram genotypes.

\section{Materials and Methods}

A field experiment was conducted involving twenty five blackgram genotypes LBG 623, LBG 752, LBG 787, LBG 806, LBG 808, LBG 818, LBG 822, LBG 828, LBG 881, LBG 884, LBG 888, LBG 951, GKB 1, GKB 2, GKB 3, GKB 4, GBG 1, VBG 13-3, VBG 14-16, TBG 125, TBG 129, TBG 104, PBG 32-2, PU 31 and TU 40in three replications at RARS, Lam. The samples that are brought for destructive sampling were oven-dried at $80^{\circ}$ for 48 hours and the dry weights of the leaves, stem, roots and pods were recorded and expressed as g plant-1. The total dry matter was calculated by adding leaves, stem, root and pod dry weights and expressed as g plant 1 .

\section{Results and Discussion}

\section{Dry matter partitioning}

Virus infection reduces plant growth by reducing dry matter accumulation. Dry matter partitioning is one of the important measures to observe the damage caused by thrips and viruses transmitted by them. The dry weights of leaf, stem, root and pod were recorded in the genotypes and were discussed hereunder.

\section{Leaf dry weight (g plant $\left.{ }^{-1}\right)$}

GBNV infection severely reduces the leaf dry weights by more than half in infected plants because the bud necrosis reduces the leaf number and size. After 30 DAS GBNV infection causes only leaf curling reducing the leaf dry weight up to $30 \%$. The leaf dry weights showed significant variation among the genotypes at 20, 40, 60 DAS and at maturity during both the seasons (Table 1).

Higher leaf dry weights were recorded in the genotypes TU 40 (5.274 g plant $^{-1}$ ) followed by LBG 884 (5.052 $\left.\mathrm{g} \mathrm{plant}^{-1}\right)$ and LBG 888 $\left(4.725 \mathrm{~g} \mathrm{plant}^{-1}\right)$ at maturity which are grouped under resistant category. Lower leaf dry weights at maturity were recorded in the genotypes GKB 4 (2.512 g plant $^{-1}$ ) under the moderately resistant category preceded by TBG 104 (2.763 g plant $\left.^{-1}\right)$ under susceptible category.

Mean leaf dry weights of resistant genotypes are $0.166,1.501,4.096$ and 4.124 g plant $^{-1}$ at 20, 40, 60 DAS and maturity respectively. The mean leaf dry weights of moderately resistant genotypes are $0.126,1.853,3.525$ and $3.861 \mathrm{~g} \mathrm{plant}^{-1}$ at 20, 40,60 DAS and maturity respectively. The mean dry weights of moderately susceptible genotypes are $0.175,1.346,3.278$ and 3.324 g plant $^{-1}$ at 20, 40,60 DAS and maturity respectively. The mean leaf dry weights of susceptible genotypes are $0.159,1.395,3.430$ and $3.471 \mathrm{~g}$ plant $^{-1}$ at 20, 40,60 DAS and maturity respectively.

Correlation studies between leaf dry weight and leaf curl disease incidence indicated that a highly significant negative correlation was 
noticed at 60 DAS $(r=-0.536)$ and maturity $(r$ $=-0.541)$ and a non-significant correlation was observed at 20 DAS and 40 DAS. Correlation of leaf dry weight with thrips population was observed to be negative and significant at 60 DAS $(r=-0.457)$ and maturity $(r=-0.467)$ and non-significant at 20 DAS and 40 DAS (Table 1).

Results obtained indicated that the leaf dry weight of susceptible genotypes was highly affected by leaf curl disease incidence and thrips population. The leaf dry weight susceptible genotypes were decreased more due to the leaf curl effect at 60 DAS and maturity. The leaf dry weight of cowpea showed a decrease due to the infection complex caused by CMV and BICMV (PioRibeiro et al., 1978).

\section{Stem dry weight (g plant $\left.{ }^{-1}\right)$}

Leaf curl disease in blackgram is caused by GBNV and its infection at initial stages of crop growth causes bud necrosis disease that reduces the stem dry weights phenomenally and leads to partial or complete yield loss. Stem dry weight showed a significant variation among the genotypes at 20, 40, 60 DAS and maturity during both the seasons (Table 2). Mean values of stem dry weights of resistant genotypes are $0.091,1.273,3.774 \mathrm{~g}$ and 3.759 g plant $^{-1}$ at 20, 40,60 DAS and at maturity respectively. The mean stem dry weights of moderately resistant genotypes are 0.076, 1.010, 2.228 and $2.450 \mathrm{~g} \mathrm{plant}^{-1}$ at 20, 40, 60 DAS and at maturity respectively.

The mean stem dry weights of moderately susceptible genotypes are $0.086,1.080,2.658$ and $2.804 \mathrm{~g} \mathrm{plant}^{-1}$ at 20, 40,60 DAS and at maturity respectively. The mean stem dry weights of susceptible genotypes are 0.075, $1.112,2.937$ and $2.790 \mathrm{~g} \mathrm{plant}^{-1}$ at 20, 40,60 DAS and at maturity respectively.
Higher stem dry weights were recorded in the genotypes TU 40 (5.032 $\left.\mathrm{g} \mathrm{plant}^{-1}\right)$ followed by TBG 129 (4.331 $\mathrm{g} \mathrm{plant}^{-1}$ ) and LBG 884 $\left(4.045 \mathrm{~g} \mathrm{plant}^{-1}\right)$ at maturity which are grouped under resistant category. Lower stem dry weights at maturity were recorded in the genotypes LBG $881 \quad\left(2.075 \quad \mathrm{~g} \quad\right.$ plant $\left.^{-1}\right)$ preceded by LBG 623 (2.216 g plant $\left.^{-1}\right)$ under the moderately resistant category.

Correlation studies of stem dry weight with leaf curl disease incidence and thrips population showed a highly significant negative correlation with both leaf curl disease incidence $(\%)$ and thrips population at 60 DAS $(r=-0.484$ and -0.530$)$ and maturity $(r=-0.549$ and -0.617$)$ respectively. The stem dry weight with leaf curl disease incidence (\%) and thrips population showed a nonsignificant negative correlation at 20 DAS and 40 DAS (Table 6).

The stem dry weights of resistant genotypes were higher when compared to any other category indicating that the resistant genotypes possess higher stem dry weights. These results indicated that the resistant genotypes were less affected by thrips and leaf curl disease. Latham et al., (2004) observed the loss of stem dry weight in lentil susceptible genotypes due to the alfalfa mosaic virus (AMV) infection. The virus infected plants showed reduced residual dry matter contents in their stems of tomato plants infected with aucuba or yellow mosaic virus (Caldwell, 1934).

\section{Root dry weight (g plant $\left.{ }^{-1}\right)$ :}

Root dry weight values varied significantly among the blackgram genotypes at 20, 40, 60 DAS and maturity during both the seasons (Table 3). Mean values of root dry weights of resistant genotypes are 0.064, 0.534, 1.346 and 1.280 g plant $^{-1}$ at 20, 40, 60 DAS and at maturity respectively. Mean root dry weights 
of moderately resistant genotypes are 0.053 , $0.469,0.981$ and $0.912 \mathrm{~g} \mathrm{plant}^{-1}$ at 20, 40, 60 DAS and at maturity respectively.

The mean root dry weights of moderately susceptible genotypes are $0.063,0.490,1.157$ and $1.083 \mathrm{~g} \mathrm{plant}^{-1}$ at 20,40,60 DAS and at maturity respectively. The mean root dry weights of susceptible genotypes are 0.060, $0.497,1.150$ and $1.081 \mathrm{~g} \mathrm{plant}^{-1}$ at 20, 40, 60 DAS and at maturity respectively.

Higher root dry weights were recorded in the genotypes TU 40 (1.485 $\mathrm{g} \mathrm{plant}^{-1}$ ) followed by LBG 884 (1.474 $\left.\mathrm{g}_{\text {plant }}{ }^{-1}\right)$ and VBG 13-3 $\left(1.411 \mathrm{~g} \mathrm{plant}^{-1}\right)$ at maturity which are grouped under resistant category. Lower root dry weights at maturity were recorded in the genotypes GKB 1 (0.705 $\left.\mathrm{g}_{\text {plant }}{ }^{-1}\right)$ under moderately resistant category preceded by GKB 3 (0.803 $\left.\mathrm{g} \mathrm{plant}^{-1}\right)$ under the resistant category.

Root dry weight not only depends on the viral infection but also the plant stature and genotypic character. It is observed that resistant genotypes have higher root dry weights. These results are in corroboration with the findings of Terry and Hahn, (1980) in cassava genotypes and Hema et al., (2015) in soybean genotypes.

\section{Pod dry weight (g plant ${ }^{-1}$ )}

Pod dry weight values showed a significant variation among the blackgram genotypes during both the seasons late rabi 2018-19 and late rabi 2019-20 at 60 DAS and maturity (Table 4).

Mean pod dry weights values of resistant genotypes are 2.159 and 3.289 g plant- 1 at 60 DAS and maturity respectively. The mean pod dry weights of moderately resistant genotypes are 1.943 and 2.698 g plant $^{-1}$ at 60 DAS and maturity respectively. The mean pod dry weights of moderately susceptible genotypes are 1.635 and $2.725 \mathrm{~g} \mathrm{plant}^{-1}$ at 60 DAS and maturity respectively. Mean pod dry weights of susceptible genotypes are 1.703 and $2.803 \mathrm{~g} \mathrm{plant}^{-1}$ at $60 \mathrm{DAS}$ and maturity respectively

Higher pod dry weights were recorded in the genotypes TU 40 (3.696 $\mathrm{g} \mathrm{plant}^{-1}$ ) followed by LBG 884 (3.538 $\mathrm{g} \mathrm{plant}^{-1}$ ) and LBG 888 (3.462 $\left.\mathrm{g} \mathrm{plant}^{-1}\right)$ at maturity which are grouped under resistant category. Lower pod dry weights at maturity were recorded in the genotypes LBG $951 \quad\left(2.479 \quad \mathrm{~g} \quad\right.$ plant $\left.^{-1}\right)$ preceded by LBG 787 (2.599 g plant $^{-1}$ ) under the moderately susceptible category.

Correlation studies indicated that pod dry weight and leaf curl disease incidence showed a highly significant negative correlation at 60 DAS $(r=-0.636)$ and maturity $(r=-0.576)$ and a similar highly significant negative correlation was obtained with thrips population at 60 DAS $(\mathrm{r}=-0.724)$ and maturity $(r=-0.670)$ (Table 6). Pod dry weight is a direct measurement parameter for yield per plant which is purely dependent on the genetic yield potential of a genotype the virus-resistant genotypes has less reduction in pod dry weight compared with the susceptible ones. These results are in acceptance with the findings of Latham et al., 2004 in CMV infected cowpea genotypes.

\section{Total dry matter (TDM) (g plant $\left.{ }^{-1}\right)$}

Total dry matter of a plant indicates the growth in terms of dry mass accumulated and it is very important to measure TDM in the viral diseases like leaf curl disease because its infection significantly reduces TDM in susceptible genotypes. A significant variation was observed among the genotypes for TDM at all the measured and TDM increased in all the genotypes from 20 DAS to maturity during both the seasons (Table 5). 
Table.1 Variability in leaf dry weight $\left(\mathrm{g}_{\text {plant }}{ }^{-1}\right.$ ) of blackgram genotypes as influenced by leaf curl disease incidence

\begin{tabular}{|c|c|c|c|c|c|c|c|c|c|c|c|c|c|}
\hline \multirow[t]{2}{*}{ S. No. } & \multirow[t]{2}{*}{ Genotypes } & \multicolumn{3}{|c|}{20 DAS } & \multicolumn{3}{|c|}{40 DAS } & \multicolumn{3}{|c|}{60 DAS } & \multicolumn{3}{|c|}{ At Maturity } \\
\hline & & $\begin{array}{c}\text { Late rabi } \\
2018-19\end{array}$ & $\begin{array}{c}\text { Late rabi } \\
2019-20\end{array}$ & $\begin{array}{c}\text { Pooled } \\
\text { mean }\end{array}$ & $\begin{array}{c}\text { Late } r a b i \\
2018-19\end{array}$ & $\begin{array}{c}\text { Late rabi } \\
2019-20\end{array}$ & $\begin{array}{c}\text { Pooled } \\
\text { mean }\end{array}$ & $\begin{array}{c}\text { Late rabi } \\
2018-19\end{array}$ & $\begin{array}{c}\text { Late rabi } \\
2019-20\end{array}$ & $\begin{array}{c}\text { Pooled } \\
\text { mean }\end{array}$ & $\begin{array}{c}\text { Late } r a b i \\
2018-19\end{array}$ & $\begin{array}{c}\text { Late rabi } \\
2019-20\end{array}$ & $\begin{array}{c}\text { Pooled } \\
\text { mean }\end{array}$ \\
\hline & Resistant & & & & & & & & & & & & \\
\hline 1 & LBG 884 & 0.233 & 0.216 & 0.225 & 1.588 & 1.496 & 1.542 & 5.340 & 4.972 & 5.156 & 5.293 & 4.811 & 5.052 \\
\hline 2 & LBG 888 & 0.199 & 0.178 & 0.188 & 1.580 & 1.398 & 1.489 & 4.942 & 4.462 & 4.702 & 4.948 & 4.503 & 4.725 \\
\hline 3 & GBG 1 & 0.169 & 0.148 & 0.159 & 1.294 & 1.128 & 1.211 & 3.585 & 3.257 & 3.421 & 3.694 & 3.237 & 3.465 \\
\hline 4 & VBG 13-3 & 0.142 & 0.113 & 0.128 & 1.341 & 1.226 & 1.284 & 3.940 & 3.700 & 3.820 & 4.010 & 3.788 & 3.899 \\
\hline 5 & TBG 129 & 0.141 & 0.108 & 0.125 & 1.827 & 1.723 & 1.775 & 3.850 & 3.425 & 3.637 & 3.948 & 3.360 & 3.654 \\
\hline 6 & PBG 32-2 & 0.193 & 0.186 & 0.190 & 1.421 & 1.319 & 1.370 & 3.629 & 3.531 & 3.580 & 3.583 & 3.609 & 3.596 \\
\hline 7 & TU 40 & 0.196 & 0.191 & 0.194 & 2.038 & 1.905 & 1.972 & 5.328 & 5.044 & 5.186 & 5.436 & 5.113 & 5.274 \\
\hline \multirow[t]{2}{*}{8} & GKB 3 & 0.133 & 0.115 & 0.124 & 1.400 & 1.331 & 1.366 & 3.404 & 3.128 & 3.266 & 3.474 & 3.173 & 3.323 \\
\hline & Mean & 0.176 & 0.157 & 0.166 & 1.561 & 1.441 & 1.501 & 4.252 & 3.940 & 4.096 & 4.298 & 3.949 & 4.124 \\
\hline \multicolumn{14}{|c|}{ Moderately resistant } \\
\hline 9 & LBG 623 & 0.151 & 0.138 & 0.145 & 1.887 & 1.681 & 1.784 & 3.528 & 3.267 & 3.398 & 3.582 & 3.210 & 3.396 \\
\hline 10 & LBG 806 & 0.143 & 0.129 & 0.136 & 1.288 & 1.185 & 1.237 & 3.102 & 2.662 & 2.882 & 3.163 & 2.808 & 2.986 \\
\hline 11 & LBG 808 & 0.178 & 0.161 & 0.170 & 1.344 & 1.199 & 1.271 & 3.079 & 2.818 & 2.948 & 3.164 & 2.841 & 3.003 \\
\hline 12 & LBG 818 & 0.168 & 0.163 & 0.165 & 1.693 & 1.570 & 1.631 & 3.641 & 2.857 & 3.249 & 3.691 & 2.904 & 3.297 \\
\hline 13 & LBG 881 & 0.152 & 0.141 & 0.147 & 1.250 & 1.084 & 1.167 & 2.745 & 2.502 & 2.624 & 2.797 & 2.506 & 2.651 \\
\hline 14 & GKB 1 & 0.175 & 0.150 & 0.162 & 1.284 & 1.188 & 1.236 & 3.055 & 2.751 & 2.903 & 3.034 & 2.854 & 2.944 \\
\hline 15 & GKB 2 & 0.143 & 0.142 & 0.143 & 1.248 & 1.250 & 1.249 & 3.137 & 2.829 & 2.983 & 3.370 & 2.718 & 3.044 \\
\hline 16 & GKB 4 & 0.161 & 0.144 & 0.153 & 1.082 & 0.893 & 0.988 & 2.709 & 2.277 & 2.493 & 2.770 & 2.254 & 2.512 \\
\hline \multirow[t]{3}{*}{17} & VBG 14-16 & 0.136 & 0.115 & 0.126 & 1.885 & 1.821 & 1.853 & 3.905 & 3.525 & 3.715 & 4.056 & 3.666 & 3.861 \\
\hline & Mean & 0.151 & 0.138 & 0.145 & 1.887 & 1.681 & 1.784 & 3.528 & 3.267 & 3.398 & 3.582 & 3.210 & 3.396 \\
\hline & \multicolumn{2}{|c|}{ Moderately susceptible } & & & & & & & & & & & \\
\hline 18 & LBG 752 & 0.252 & 0.231 & 0.241 & 1.785 & 1.654 & 1.720 & 3.723 & 3.474 & 3.598 & 3.722 & 3.557 & 3.640 \\
\hline 19 & LBG 787 & 0.169 & 0.150 & 0.160 & 1.642 & 1.456 & 1.549 & 3.658 & 3.267 & 3.462 & 3.669 & 3.383 & 3.526 \\
\hline 20 & LBG 822 & 0.138 & 0.127 & 0.133 & 1.307 & 1.138 & 1.222 & 3.590 & 3.193 & 3.392 & 3.576 & 3.168 & 3.372 \\
\hline 21 & LBG 828 & 0.182 & 0.155 & 0.169 & 1.098 & 1.049 & 1.074 & 3.266 & 2.905 & 3.086 & 3.244 & 3.016 & 3.130 \\
\hline 22 & LBG 951 & 0.178 & 0.151 & 0.164 & 1.273 & 1.164 & 1.219 & 2.936 & 2.699 & 2.817 & 3.055 & 2.772 & 2.913 \\
\hline \multirow[t]{3}{*}{23} & PU 31 & 0.188 & 0.178 & 0.183 & 1.370 & 1.221 & 1.296 & 3.446 & 3.179 & 3.313 & 3.489 & 3.233 & 3.361 \\
\hline & Mean & 0.185 & 0.165 & 0.175 & 1.413 & 1.280 & 1.346 & 3.436 & 3.119 & 3.278 & 3.459 & 3.188 & 3.324 \\
\hline & Susceptible & & & & & & & & & & & & \\
\hline 24 & TBG 125 & 0.138 & 0.129 & 0.133 & 1.296 & 1.196 & 1.246 & 3.510 & 3.240 & 3.375 & 3.736 & 3.032 & 3.384 \\
\hline \multirow[t]{2}{*}{25} & TBG 104 & 0.133 & 0.107 & 0.120 & 1.170 & 1.096 & 1.133 & 2.979 & 2.501 & 2.740 & 2.969 & 2.557 & 2.763 \\
\hline & Mean & 0.136 & 0.118 & 0.127 & 1.233 & 1.146 & 1.190 & 3.245 & 2.871 & 3.058 & 3.352 & 2.794 & 3.073 \\
\hline \multicolumn{2}{|c|}{ Grand mean } & 0.168 & 0.151 & 0.159 & 1.456 & 1.335 & 1.395 & 3.601 & 3.259 & 3.430 & 3.659 & 3.283 & 3.471 \\
\hline \multicolumn{2}{|c|}{$\mathrm{SE}(\mathrm{m}) \pm$} & 0.01 & 0.00 & 0.00 & 0.03 & 0.04 & 0.03 & 0.05 & 0.05 & 0.04 & 0.08 & 0.08 & 0.06 \\
\hline \multicolumn{2}{|c|}{$\mathrm{CD}(0.05)$} & 0.02 & 0.01 & 0.01 & 0.09 & 0.11 & 0.08 & 0.15 & 0.13 & 0.11 & 0.22 & 0.22 & 0.17 \\
\hline \multicolumn{2}{|c|}{ CV $(\%)$} & 7.87 & 3.64 & 5.00 & 3.68 & 4.80 & 3.33 & 2.58 & 2.47 & 1.90 & 3.70 & 3.99 & 3.01 \\
\hline
\end{tabular}


Table. 2 Variability in stem dry weight $\left(\mathrm{g} \mathrm{plant}^{-1}\right.$ ) of Blackgram genotypes as influenced by leaf curl disease incidence

\begin{tabular}{|c|c|c|c|c|c|c|c|c|c|c|c|c|c|}
\hline \multirow[t]{2}{*}{ S. No. } & \multirow[t]{2}{*}{ Genotypes } & \multicolumn{3}{|c|}{20 DAS } & \multicolumn{3}{|c|}{40 DAS } & \multicolumn{3}{|c|}{60 DAS } & \multicolumn{3}{|c|}{ At Maturity } \\
\hline & & $\begin{array}{c}\text { Late rabi } \\
2018-19\end{array}$ & $\begin{array}{c}\text { Late rabi } \\
2019-20\end{array}$ & $\begin{array}{c}\text { Pooled } \\
\text { mean }\end{array}$ & $\begin{array}{c}\text { Late rabi } \\
2018-19\end{array}$ & $\begin{array}{c}\text { Late rabi } \\
2019-20\end{array}$ & $\begin{array}{c}\text { Pooled } \\
\text { mean }\end{array}$ & $\begin{array}{c}\text { Late rabi } \\
2018-19\end{array}$ & $\begin{array}{c}\text { Late rabi } \\
2019-20\end{array}$ & $\begin{array}{c}\text { Pooled } \\
\text { mean }\end{array}$ & $\begin{array}{c}\text { Late } r a b i \\
2018-19\end{array}$ & $\begin{array}{c}\text { Late rabi } \\
2019-20\end{array}$ & $\begin{array}{c}\text { Pooled } \\
\text { mean }\end{array}$ \\
\hline & Resistant & & & & & & & & & & & & \\
\hline 1 & LBG 884 & 0.105 & 0.084 & 0.095 & 1.537 & 1.428 & 1.483 & 4.170 & 3.751 & 3.961 & 4.261 & 3.828 & 4.045 \\
\hline 2 & LBG 888 & 0.123 & 0.099 & 0.111 & 1.136 & 1.028 & 1.082 & 3.496 & 3.293 & 3.394 & 3.406 & 3.259 & 3.332 \\
\hline 3 & GBG 1 & 0.100 & 0.083 & 0.092 & 0.951 & 0.918 & 0.935 & 2.587 & 2.665 & 2.626 & 3.160 & 3.188 & 3.174 \\
\hline 4 & VBG 13-3 & 0.106 & 0.079 & 0.092 & 1.179 & 1.062 & 1.120 & 3.447 & 3.258 & 3.353 & 3.596 & 3.446 & 3.521 \\
\hline 5 & TBG 129 & 0.089 & 0.077 & 0.083 & 1.427 & 1.339 & 1.383 & 4.580 & 4.288 & 4.434 & 4.523 & 4.138 & 4.331 \\
\hline 6 & PBG 32-2 & 0.097 & 0.073 & 0.085 & 1.315 & 1.202 & 1.259 & 4.288 & 4.174 & 4.231 & 3.799 & 3.908 & 3.854 \\
\hline 7 & TU 40 & 0.091 & 0.085 & 0.088 & 1.688 & 1.579 & 1.634 & 5.250 & 5.060 & 5.155 & 5.135 & 4.928 & 5.032 \\
\hline \multirow[t]{2}{*}{8} & GKB 3 & 0.090 & 0.078 & 0.084 & 1.344 & 1.223 & 1.284 & 3.220 & 2.859 & 3.040 & 2.936 & 2.634 & 2.785 \\
\hline & Mean & 0.100 & 0.082 & 0.091 & 1.322 & 1.222 & 1.273 & 3.880 & 3.669 & 3.774 & 3.852 & 3.666 & 3.759 \\
\hline \multicolumn{14}{|c|}{ Moderately resistant } \\
\hline 9 & LBG 623 & 0.105 & 0.096 & 0.101 & 1.120 & 1.027 & 1.074 & 2.118 & 2.060 & 2.089 & 2.284 & 2.148 & 2.216 \\
\hline 10 & LBG 806 & 0.073 & 0.062 & 0.068 & 0.923 & 0.813 & 0.868 & 2.262 & 1.863 & 2.062 & 2.471 & 2.205 & 2.338 \\
\hline 11 & LBG 808 & 0.090 & 0.063 & 0.077 & 0.986 & 0.848 & 0.917 & 2.328 & 2.084 & 2.206 & 2.721 & 2.507 & 2.614 \\
\hline 12 & LBG 818 & 0.090 & 0.076 & 0.083 & 1.399 & 1.304 & 1.352 & 3.058 & 2.429 & 2.743 & 3.321 & 2.793 & 3.057 \\
\hline 13 & LBG 881 & 0.079 & 0.058 & 0.068 & 0.894 & 0.803 & 0.848 & 1.992 & 1.897 & 1.944 & 2.125 & 2.024 & 2.075 \\
\hline 14 & GKB 1 & 0.070 & 0.062 & 0.066 & 0.922 & 0.824 & 0.873 & 2.128 & 1.896 & 2.012 & 2.457 & 2.335 & 2.396 \\
\hline 15 & GKB 2 & 0.081 & 0.065 & 0.073 & 0.992 & 0.863 & 0.927 & 2.447 & 1.958 & 2.203 & 2.364 & 2.076 & 2.220 \\
\hline 16 & GKB 4 & 0.073 & 0.065 & 0.069 & 0.821 & 0.697 & 0.759 & 1.989 & 1.752 & 1.871 & 2.507 & 2.193 & 2.350 \\
\hline \multirow[t]{3}{*}{17} & VBG 14-16 & 0.089 & 0.064 & 0.077 & 1.528 & 1.413 & 1.471 & 3.127 & 2.722 & 2.924 & 2.957 & 2.612 & 2.785 \\
\hline & Mean & 0.083 & 0.068 & 0.076 & 1.065 & 0.955 & 1.010 & 2.383 & 2.073 & 2.228 & 2.579 & 2.321 & 2.450 \\
\hline & \multicolumn{2}{|c|}{ Moderately susceptible } & & & & & & & & & & & \\
\hline 18 & LBG 752 & 0.109 & 0.108 & 0.109 & 1.405 & 1.306 & 1.356 & 2.893 & 2.753 & 2.823 & 3.005 & 2.952 & 2.979 \\
\hline 19 & LBG 787 & 0.088 & 0.080 & 0.084 & 1.112 & 1.009 & 1.060 & 2.460 & 2.273 & 2.366 & 2.585 & 2.455 & 2.520 \\
\hline 20 & LBG 822 & 0.078 & 0.061 & 0.070 & 1.149 & 1.038 & 1.094 & 3.124 & 2.950 & 3.037 & 2.955 & 2.792 & 2.874 \\
\hline 21 & LBG 828 & 0.093 & 0.064 & 0.079 & 0.933 & 0.820 & 0.876 & 2.843 & 2.402 & 2.622 & 2.944 & 2.747 & 2.845 \\
\hline 22 & LBG 951 & 0.093 & 0.086 & 0.089 & 1.045 & 0.955 & 1.000 & 2.415 & 2.244 & 2.329 & 2.806 & 2.631 & 2.719 \\
\hline \multirow[t]{3}{*}{23} & PU 31 & 0.094 & 0.074 & 0.084 & 1.160 & 1.025 & 1.093 & 2.871 & 2.672 & 2.772 & 2.995 & 2.781 & 2.888 \\
\hline & Mean & 0.093 & 0.079 & 0.086 & 1.134 & 1.026 & 1.080 & 2.768 & 2.549 & 2.658 & 2.882 & 2.726 & 2.804 \\
\hline & Susceptible & & & & & & & & & & & & \\
\hline 24 & TBG 125 & 0.070 & 0.073 & 0.072 & 1.070 & 0.994 & 1.032 & 2.690 & 2.559 & 2.624 & 2.970 & 2.424 & 2.697 \\
\hline \multirow[t]{2}{*}{25} & TBG 104 & 0.082 & 0.074 & 0.078 & 1.087 & 0.943 & 1.015 & 3.465 & 3.034 & 3.250 & 3.099 & 2.668 & 2.883 \\
\hline & Mean & 0.076 & 0.074 & 0.075 & 1.079 & 0.969 & 1.024 & 3.078 & 2.797 & 2.937 & 3.035 & 2.546 & 2.790 \\
\hline \multicolumn{2}{|r|}{ Grand mean } & 0.090 & 0.076 & 0.083 & 1.165 & 1.058 & 1.112 & 3.010 & 2.756 & 2.883 & 3.095 & 2.867 & 2.981 \\
\hline \multicolumn{2}{|r|}{$\mathrm{SE}(\mathbf{m}) \pm$} & 0.006 & 0.005 & 0.004 & 0.048 & 0.034 & 0.032 & 0.035 & 0.038 & 0.027 & 0.064 & 0.060 & 0.048 \\
\hline \multicolumn{2}{|r|}{$\mathrm{CD}(0.05)$} & 0.016 & 0.014 & 0.012 & 0.137 & 0.098 & 0.092 & 0.101 & 0.107 & 0.077 & 0.182 & 0.172 & 0.137 \\
\hline \multicolumn{2}{|r|}{ CV $(\%)$} & 10.97 & 10.98 & 8.61 & 7.14 & 5.64 & 5.02 & 2.04 & 2.38 & 1.64 & 3.57 & 3.65 & 2.80 \\
\hline
\end{tabular}


Table.3 Variability in root dry weight $\left(\mathrm{g}_{\text {plant }}{ }^{-1}\right.$ ) of blackgram genotypes as influenced by leaf curl disease incidence

\begin{tabular}{|c|c|c|c|c|c|c|c|c|c|c|c|c|c|}
\hline \multirow{2}{*}{$\begin{array}{l}\text { S. } \\
\text { No. }\end{array}$} & \multirow[t]{2}{*}{ Genotypes } & \multicolumn{3}{|c|}{20 DAS } & \multicolumn{3}{|c|}{40 DAS } & \multicolumn{3}{|c|}{60 DAS } & \multicolumn{3}{|c|}{ At Maturity } \\
\hline & & $\begin{array}{c}\text { Late } r a b i \\
2018-19\end{array}$ & $\begin{array}{c}\text { Late rabi } \\
2019-20\end{array}$ & $\begin{array}{c}\text { Pooled } \\
\text { mean }\end{array}$ & $\begin{array}{l}\text { Late } r a b i \\
2018-19\end{array}$ & $\begin{array}{c}\text { Late rabi } \\
2019-20\end{array}$ & $\begin{array}{c}\text { Pooled } \\
\text { mean }\end{array}$ & $\begin{array}{c}\text { Late rabi } \\
\text { 2018-19 }\end{array}$ & $\begin{array}{c}\text { Late rabi } \\
2019-20\end{array}$ & $\begin{array}{c}\text { Pooled } \\
\text { mean }\end{array}$ & $\begin{array}{l}\text { Late } r a b i \\
2018-19\end{array}$ & $\begin{array}{c}\text { Late rabi } \\
2019-20\end{array}$ & $\begin{array}{c}\text { Pooled } \\
\text { mean }\end{array}$ \\
\hline & Resistant & & & & & & & & & & & & \\
\hline 1 & LBG 884 & 0.065 & 0.061 & 0.063 & 0.617 & 0.508 & 0.562 & 1.660 & 1.421 & 1.541 & 1.604 & 1.343 & 1.474 \\
\hline 2 & LBG 888 & 0.061 & 0.056 & 0.058 & 0.574 & 0.473 & 0.523 & 1.386 & 1.218 & 1.302 & 1.348 & 1.140 & 1.244 \\
\hline 3 & GBG 1 & 0.051 & 0.047 & 0.049 & 0.478 & 0.377 & 0.427 & 1.157 & 1.061 & 1.109 & 1.093 & 0.987 & 1.040 \\
\hline 4 & VBG 13-3 & 0.078 & 0.070 & 0.074 & 0.559 & 0.453 & 0.506 & 1.562 & 1.390 & 1.476 & 1.496 & 1.327 & 1.411 \\
\hline 5 & TBG 129 & 0.056 & 0.047 & 0.052 & 0.636 & 0.499 & 0.567 & 1.549 & 1.402 & 1.476 & 1.473 & 1.318 & 1.395 \\
\hline 6 & PBG 32-2 & 0.082 & 0.075 & 0.079 & 0.602 & 0.511 & 0.557 & 1.525 & 1.392 & 1.458 & 1.458 & 1.315 & 1.387 \\
\hline 7 & TU 40 & 0.072 & 0.061 & 0.067 & 0.759 & 0.673 & 0.716 & 1.613 & 1.471 & 1.542 & 1.582 & 1.388 & 1.485 \\
\hline \multirow[t]{2}{*}{8} & GKB 3 & 0.072 & 0.067 & 0.069 & 0.485 & 0.347 & 0.416 & 0.976 & 0.751 & 0.864 & 0.932 & 0.675 & 0.803 \\
\hline & Mean & 0.067 & 0.061 & 0.064 & 0.589 & 0.480 & 0.534 & 1.429 & 1.263 & 1.346 & 1.373 & 1.187 & 1.280 \\
\hline \multicolumn{14}{|c|}{ Moderately resistant } \\
\hline 9 & LBG 623 & 0.069 & 0.060 & 0.064 & 0.613 & 0.511 & 0.562 & 1.028 & 0.878 & 0.953 & 0.968 & 0.803 & 0.885 \\
\hline 10 & LBG 806 & 0.059 & 0.049 & 0.054 & 0.494 & 0.378 & 0.436 & 1.115 & 0.956 & 1.035 & 1.060 & 0.889 & 0.975 \\
\hline 11 & LBG 808 & 0.062 & 0.052 & 0.057 & 0.427 & 0.321 & 0.374 & 0.978 & 0.792 & 0.885 & 0.888 & 0.734 & 0.811 \\
\hline 12 & LBG 818 & 0.046 & 0.037 & 0.042 & 0.617 & 0.528 & 0.573 & 1.104 & 0.910 & 1.007 & 1.033 & 0.836 & 0.934 \\
\hline 13 & LBG 881 & 0.036 & 0.034 & 0.035 & 0.538 & 0.418 & 0.478 & 1.148 & 1.079 & 1.113 & 1.070 & 1.005 & 1.038 \\
\hline 14 & GKB 1 & 0.054 & 0.051 & 0.053 & 0.414 & 0.310 & 0.362 & 0.855 & 0.695 & 0.775 & 0.786 & 0.624 & 0.705 \\
\hline 15 & GKB 2 & 0.056 & 0.052 & 0.054 & 0.469 & 0.379 & 0.424 & 1.033 & 0.847 & 0.940 & 0.983 & 0.779 & 0.881 \\
\hline 16 & GKB 4 & 0.063 & 0.056 & 0.060 & 0.450 & 0.358 & 0.404 & 1.002 & 0.989 & 0.995 & 0.934 & 0.906 & 0.920 \\
\hline \multirow[t]{2}{*}{17} & VBG 14-16 & 0.062 & 0.052 & 0.057 & 0.651 & 0.563 & 0.607 & 1.236 & 1.015 & 1.125 & 1.186 & 0.935 & 1.061 \\
\hline & Mean & 0.056 & 0.049 & 0.053 & 0.519 & 0.418 & 0.469 & 1.055 & 0.907 & 0.981 & 0.990 & 0.835 & 0.912 \\
\hline 18 & LBG 752 & 0.080 & 0.071 & 0.076 & 0.684 & 0.542 & 0.613 & 1.400 & 1.201 & 1.300 & 1.330 & 1.126 & 1.228 \\
\hline 19 & LBG 787 & 0.078 & 0.068 & 0.073 & 0.584 & 0.393 & 0.489 & 1.176 & 1.026 & 1.101 & 1.110 & 0.957 & 1.033 \\
\hline 20 & LBG 822 & 0.051 & 0.042 & 0.047 & 0.561 & 0.398 & 0.480 & 1.449 & 1.243 & 1.346 & 1.391 & 1.158 & 1.275 \\
\hline 21 & LBG 828 & 0.079 & 0.071 & 0.075 & 0.450 & 0.331 & 0.390 & 1.051 & 0.890 & 0.971 & 0.971 & 0.816 & 0.893 \\
\hline 22 & LBG 951 & 0.057 & 0.050 & 0.054 & 0.511 & 0.355 & 0.433 & 1.160 & 0.935 & 1.047 & 1.081 & 0.860 & 0.971 \\
\hline \multirow[t]{2}{*}{23} & PU 31 & 0.087 & 0.078 & 0.083 & 0.589 & 0.480 & 0.534 & 1.244 & 1.105 & 1.175 & 1.167 & 1.025 & 1.096 \\
\hline & Mean & 0.072 & 0.063 & 0.068 & 0.563 & 0.417 & 0.490 & 1.247 & 1.067 & 1.157 & 1.175 & 0.990 & 1.083 \\
\hline 24 & TBG 125 & 0.055 & 0.048 & 0.052 & 0.590 & 0.445 & 0.517 & 1.347 & 1.146 & 1.247 & 1.294 & 1.077 & 1.186 \\
\hline \multirow[t]{2}{*}{25} & TBG 104 & 0.065 & 0.055 & 0.060 & 0.533 & 0.419 & 0.476 & 1.049 & 0.897 & 0.973 & 0.986 & 0.818 & 0.902 \\
\hline & Mean & 0.060 & 0.052 & 0.056 & 0.562 & 0.432 & 0.497 & 1.198 & 1.022 & 1.110 & 1.140 & 0.948 & 1.044 \\
\hline & Grand mean & 0.064 & 0.056 & 0.060 & 0.555 & 0.439 & 0.497 & 1.232 & 1.068 & 1.150 & 1.169 & 0.994 & 1.081 \\
\hline & SE $(m) \pm$ & 0.004 & 0.003 & 0.003 & 0.019 & 0.021 & 0.016 & 0.056 & 0.067 & 0.050 & 0.042 & 0.069 & 0.044 \\
\hline & $\mathrm{CD}(0.05)$ & 0.012 & 0.009 & 0.009 & 0.053 & 0.059 & 0.046 & 0.158 & 0.192 & 0.142 & 0.120 & 0.197 & 0.125 \\
\hline & $\mathrm{CV}(\%)$ & 11.63 & 9.62 & 9.61 & 5.84 & 8.24 & 5.62 & 7.83 & 10.93 & 7.51 & 6.27 & 12.11 & 7.05 \\
\hline
\end{tabular}


Table.4 Variability in pod dry weight $\left(\mathrm{g} \mathrm{plant}^{-1}\right)$ of blackgram genotypes as influenced by leaf curl disease incidence

\begin{tabular}{|c|c|c|c|c|c|c|c|}
\hline \multirow{2}{*}{$\begin{array}{l}\text { S. } \\
\text { No. }\end{array}$} & \multirow[t]{2}{*}{ Genotypes } & \multicolumn{3}{|c|}{60 DAS } & \multicolumn{3}{|c|}{ At Maturity } \\
\hline & & $\begin{array}{c}\text { Late } \\
\text { rabi } \\
\text { 2018-19 }\end{array}$ & $\begin{array}{c}\text { Late } \\
\text { rabi } \\
2019-20\end{array}$ & $\begin{array}{c}\text { Pooled } \\
\text { mean }\end{array}$ & $\begin{array}{c}\text { Late } \\
\text { rabi } \\
\text { 2018-19 }\end{array}$ & $\begin{array}{c}\text { Late } \\
\text { rabi } \\
2019-20\end{array}$ & $\begin{array}{c}\text { Pooled } \\
\text { mean }\end{array}$ \\
\hline & Resistant & & & & & & \\
\hline 1 & LBG 884 & 1.882 & 1.712 & 1.797 & 3.685 & 3.391 & 3.538 \\
\hline 2 & LBG 888 & 2.101 & 1.897 & 1.999 & 3.647 & 3.277 & 3.462 \\
\hline 3 & GBG 1 & 2.267 & 2.004 & 2.135 & 3.088 & 2.811 & 2.950 \\
\hline 4 & VBG 13-3 & 2.334 & 2.006 & 2.170 & 3.254 & 2.993 & 3.123 \\
\hline 5 & TBG 129 & 2.435 & 2.214 & 2.324 & 3.541 & 3.278 & 3.410 \\
\hline 6 & PBG 32-2 & 2.438 & 2.208 & 2.323 & 3.361 & 3.097 & 3.229 \\
\hline 7 & TU 40 & 2.647 & 2.399 & 2.523 & 3.825 & 3.566 & 3.696 \\
\hline \multirow[t]{2}{*}{8} & GKB 3 & 2.110 & 1.898 & 2.004 & 3.025 & 2.786 & 2.906 \\
\hline & Mean & 2.277 & 2.042 & 2.159 & 3.428 & 3.150 & 3.289 \\
\hline \multicolumn{8}{|c|}{ Moderately resistant } \\
\hline 9 & LBG 623 & 1.311 & 1.075 & 1.193 & 2.839 & 2.559 & 2.699 \\
\hline 10 & LBG 806 & 1.899 & 1.610 & 1.754 & 3.293 & 2.884 & 3.089 \\
\hline 11 & LBG 808 & 1.883 & 1.606 & 1.745 & 3.304 & 3.066 & 3.185 \\
\hline 12 & LBG 818 & 1.612 & 1.427 & 1.519 & 2.839 & 2.601 & 2.720 \\
\hline 13 & LBG 881 & 2.167 & 1.951 & 2.059 & 3.428 & 3.215 & 3.321 \\
\hline 14 & GKB 1 & 2.003 & 1.730 & 1.866 & 3.058 & 2.795 & 2.926 \\
\hline 15 & GKB 2 & 1.954 & 1.725 & 1.839 & 2.601 & 2.444 & 2.522 \\
\hline 16 & GKB 4 & 1.787 & 1.440 & 1.614 & 2.803 & 2.549 & 2.676 \\
\hline 17 & VBG 14-16 & 2.105 & 1.780 & 1.943 & 2.811 & 2.546 & 2.678 \\
\hline \multicolumn{8}{|c|}{ Moderately susceptible } \\
\hline & Mean & 1.858 & 1.594 & 1.726 & 2.997 & 2.740 & 2.868 \\
\hline 18 & LBG 752 & 1.860 & 1.678 & 1.769 & 2.933 & 2.673 & 2.803 \\
\hline 19 & LBG 787 & 1.825 & 1.534 & 1.679 & 2.730 & 2.468 & 2.599 \\
\hline 20 & LBG 822 & 1.584 & 1.329 & 1.457 & 2.839 & 2.572 & 2.706 \\
\hline 21 & LBG 828 & 1.586 & 1.373 & 1.479 & 3.223 & 2.980 & 3.101 \\
\hline 22 & LBG 951 & 1.895 & 1.464 & 1.680 & 2.610 & 2.349 & 2.479 \\
\hline \multirow[t]{3}{*}{23} & PU 31 & 1.911 & 1.585 & 1.748 & 2.787 & 2.541 & 2.664 \\
\hline & Mean & 1.777 & 1.494 & 1.635 & 2.854 & 2.597 & 2.725 \\
\hline & Susceptible & & & & & & \\
\hline 24 & TBG 125 & 1.799 & 1.521 & 1.660 & 2.868 & 2.823 & 2.845 \\
\hline \multirow[t]{2}{*}{25} & TBG 104 & 1.996 & 1.495 & 1.745 & 2.896 & 2.627 & 2.761 \\
\hline & Mean & 1.898 & 1.508 & 1.703 & 2.882 & 2.725 & 2.803 \\
\hline \multicolumn{2}{|r|}{ Grand Mean } & 1.976 & 1.706 & 1.841 & 3.091 & 2.836 & 2.964 \\
\hline & $\mathrm{SE}(\mathrm{m}) \pm$ & 0.043 & 0.042 & 0.033 & 0.062 & 0.090 & 0.069 \\
\hline & $\mathrm{CD}(0.05)$ & 0.123 & 0.120 & 0.094 & 0.177 & 0.256 & 0.195 \\
\hline & $\mathrm{CV}(\%)$ & 3.79 & 4.30 & 3.09 & 3.48 & 5.50 & 4.01 \\
\hline
\end{tabular}


Table.5 Variability in total dry weight $\left(\mathrm{g}_{\mathrm{g}}\right.$ plant $^{-1}$ ) of Blackgram genotypes as influenced by leaf curl disease incidence

\begin{tabular}{|c|c|c|c|c|c|c|c|c|c|c|c|c|c|}
\hline \multirow{2}{*}{$\begin{array}{l}\text { S. } \\
\text { No. }\end{array}$} & \multirow[t]{2}{*}{ Genotypes } & \multicolumn{3}{|c|}{20 DAS } & \multicolumn{3}{|c|}{40 DAS } & \multicolumn{3}{|c|}{60 DAS } & \multicolumn{3}{|c|}{ At Maturity } \\
\hline & & $\begin{array}{l}\text { Late } r a b i \\
2018-19\end{array}$ & $\begin{array}{c}\text { Late rabi } \\
2019-20\end{array}$ & $\begin{array}{c}\text { Pooled } \\
\text { mean }\end{array}$ & $\begin{array}{c}\text { Late rabi } \\
2018-19\end{array}$ & $\begin{array}{c}\text { Late rabi } \\
2019-20\end{array}$ & $\begin{array}{c}\text { Pooled } \\
\text { mean }\end{array}$ & $\begin{array}{c}\text { Late rabi } \\
\text { 2018-19 }\end{array}$ & $\begin{array}{c}\text { Late rabi } \\
2019-20\end{array}$ & $\begin{array}{c}\text { Pooled } \\
\text { mean }\end{array}$ & $\begin{array}{c}\text { Late rabi } \\
\text { 2018-19 }\end{array}$ & $\begin{array}{c}\text { Late rabi } \\
2019-20\end{array}$ & $\begin{array}{c}\text { Pooled } \\
\text { mean }\end{array}$ \\
\hline & Resistant & & & & & & & & & & & & \\
\hline 1 & LBG 884 & 0.40 & 0.36 & 0.38 & 3.74 & 3.43 & 3.59 & 13.05 & 11.86 & 12.45 & 14.84 & 13.37 & 14.11 \\
\hline 2 & LBG 888 & 0.38 & 0.33 & 0.36 & 3.29 & 2.90 & 3.09 & 11.93 & 10.87 & 11.40 & 13.35 & 12.18 & 12.76 \\
\hline 3 & GBG 1 & 0.32 & 0.28 & 0.30 & 2.72 & 2.42 & 2.57 & 9.60 & 8.99 & 9.29 & 11.04 & 10.22 & 10.63 \\
\hline 4 & VBG 13-3 & 0.33 & 0.26 & 0.29 & 3.08 & 2.74 & 2.91 & 11.28 & 10.35 & 10.82 & 12.36 & 11.55 & 11.95 \\
\hline 5 & TBG 129 & 0.29 & 0.23 & 0.26 & 3.89 & 3.56 & 3.73 & 12.41 & 11.33 & 11.87 & 13.48 & 12.09 & 12.79 \\
\hline 6 & PBG 32-2 & 0.37 & 0.33 & 0.35 & 3.34 & 3.03 & 3.19 & 11.88 & 11.31 & 11.59 & 12.20 & 11.93 & 12.06 \\
\hline 7 & TU 40 & 0.36 & 0.34 & 0.35 & 4.49 & 4.16 & 4.32 & 14.84 & 13.97 & 14.41 & 15.98 & 15.00 & 15.49 \\
\hline \multirow[t]{2}{*}{8} & GKB 3 & 0.29 & 0.26 & 0.28 & 3.23 & 2.90 & 3.07 & 9.71 & 8.64 & 9.17 & 10.37 & 9.27 & 9.82 \\
\hline & Mean & 0.34 & 0.30 & 0.32 & 3.47 & 3.14 & 3.31 & 11.84 & 10.91 & 11.38 & 12.95 & 11.95 & 12.45 \\
\hline \multicolumn{14}{|c|}{ Moderately resistant } \\
\hline 9 & LBG 623 & 0.33 & 0.29 & 0.31 & 3.62 & 3.22 & 3.42 & 7.98 & 7.28 & 7.63 & 9.67 & 8.72 & 9.20 \\
\hline 10 & LBG 806 & 0.28 & 0.24 & 0.26 & 2.70 & 2.38 & 2.54 & 8.38 & 7.09 & 7.73 & 9.99 & 8.79 & 9.39 \\
\hline 11 & LBG 808 & 0.33 & 0.28 & 0.30 & 2.76 & 2.37 & 2.56 & 8.27 & 7.30 & 7.78 & 10.08 & 9.15 & 9.61 \\
\hline 12 & LBG 818 & 0.30 & 0.28 & 0.29 & 3.71 & 3.40 & 3.56 & 9.41 & 7.62 & 8.52 & 10.88 & 9.13 & 10.01 \\
\hline 13 & LBG 881 & 0.27 & 0.23 & 0.25 & 2.68 & 2.30 & 2.49 & 8.05 & 7.43 & 7.74 & 9.42 & 8.75 & 9.08 \\
\hline 14 & GKB 1 & 0.30 & 0.26 & 0.28 & 2.62 & 2.32 & 2.47 & 8.04 & 7.07 & 7.56 & 9.33 & 8.61 & 8.97 \\
\hline 15 & GKB 2 & 0.28 & 0.26 & 0.27 & 2.71 & 2.49 & 2.60 & 8.57 & 7.36 & 7.97 & 9.32 & 8.02 & 8.67 \\
\hline 16 & GKB 4 & 0.30 & 0.27 & 0.28 & 2.35 & 1.95 & 2.15 & 7.49 & 6.46 & 6.97 & 9.01 & 7.90 & 8.46 \\
\hline \multirow[t]{3}{*}{17} & VBG 14-16 & 0.29 & 0.23 & 0.26 & 4.06 & 3.80 & 3.93 & 10.37 & 9.04 & 9.71 & 11.01 & 9.76 & 10.38 \\
\hline & Mean & 0.30 & 0.26 & 0.28 & 3.02 & 2.69 & 2.86 & 8.51 & 7.41 & 7.96 & 9.86 & 8.76 & 9.31 \\
\hline & \multicolumn{2}{|c|}{ Moderately Susceptible } & & & & & & & & & & & \\
\hline 18 & LBG 752 & 0.44 & 0.41 & 0.43 & 3.87 & 3.50 & 3.69 & 9.88 & 9.11 & 9.49 & 10.99 & 10.31 & 10.65 \\
\hline 19 & LBG 787 & 0.34 & 0.30 & 0.32 & 3.34 & 2.86 & 3.10 & 9.12 & 8.10 & 8.61 & 10.09 & 9.26 & 9.68 \\
\hline 20 & LBG 822 & 0.27 & 0.23 & 0.25 & 3.02 & 2.57 & 2.80 & 9.75 & 8.72 & 9.23 & 10.76 & 9.69 & 10.23 \\
\hline 21 & LBG 828 & 0.35 & 0.29 & 0.32 & 2.48 & 2.20 & 2.34 & 8.75 & 7.57 & 8.16 & 10.38 & 9.56 & 9.97 \\
\hline 22 & LBG 951 & 0.33 & 0.29 & 0.31 & 2.83 & 2.47 & 2.65 & 8.41 & 7.34 & 7.87 & 9.55 & 8.61 & 9.08 \\
\hline \multirow[t]{3}{*}{23} & PU 31 & 0.37 & 0.33 & 0.35 & 3.12 & 2.73 & 2.92 & 9.47 & 8.54 & 9.01 & 10.44 & 9.58 & 10.01 \\
\hline & Mean & 0.35 & 0.31 & 0.33 & 3.11 & 2.72 & 2.92 & 9.23 & 8.23 & 8.73 & 10.37 & 9.50 & 9.94 \\
\hline & Susceptible & & & & & & & & & & & & \\
\hline 24 & TBG 125 & 0.26 & 0.25 & 0.26 & 2.96 & 2.64 & 2.80 & 9.35 & 8.47 & 8.91 & 10.87 & 9.36 & 10.11 \\
\hline \multirow[t]{2}{*}{25} & TBG 104 & 0.28 & 0.24 & 0.26 & 2.79 & 2.46 & 2.62 & 9.49 & 7.93 & 8.71 & 9.95 & 8.67 & 9.31 \\
\hline & Mean & 0.27 & 0.24 & 0.26 & 2.87 & 2.55 & 2.71 & 9.42 & 8.20 & 8.81 & 10.41 & 9.01 & 9.71 \\
\hline \multicolumn{2}{|r|}{$\mathrm{SE}(\mathbf{m}) \pm$} & 0.01 & 0.01 & 0.01 & 0.07 & 0.06 & 0.05 & 0.11 & 0.13 & 0.09 & 0.16 & 0.16 & 0.13 \\
\hline \multicolumn{2}{|r|}{$\mathrm{CD}(0.05)$} & 0.03 & 0.02 & 0.02 & 0.19 & 0.17 & 0.14 & 0.31 & 0.37 & 0.27 & 0.45 & 0.45 & 0.36 \\
\hline \multicolumn{2}{|r|}{ CV(\%) } & 5.99 & 4.53 & 4.58 & 3.60 & 3.56 & 2.82 & 1.89 & 2.60 & 1.75 & 2.51 & 2.72 & 2.11 \\
\hline
\end{tabular}


Table.6 Correlations of dry matter partitioning with leaf curl disease incidence and thrips population in blackgram

\begin{tabular}{|c|c|c|c|c|c|}
\hline & Parameter & 20 DAS & 40 DAS & 60 DAS & Maturity \\
\hline Disease incidence & \multirow{2}{*}{$\begin{array}{c}\text { Leaf dry } \\
\text { weight }\end{array}$} & $-0.161^{\mathrm{NS}}$ & $-0.298^{\mathrm{NS}}$ & $-0.536 * *$ & $-0.541 * *$ \\
\hline Thrips population & & $-0.195^{\mathrm{NS}}$ & $-0.066^{\mathrm{NS}}$ & $-0.457 *$ & $-0.467^{*}$ \\
\hline Disease incidence & \multirow{2}{*}{$\begin{array}{c}\text { Stem dry } \\
\text { weight }\end{array}$} & $-0.277^{\mathrm{NS}}$ & $-0.358^{\mathrm{NS}}$ & $-0.484 *$ & $-0.549 * *$ \\
\hline Thrips population & & $0.004^{\mathrm{NS}}$ & $-0.301^{\mathrm{NS}}$ & $-0.530 * *$ & $-0.617 * *$ \\
\hline Disease incidence & \multirow{2}{*}{ pod dry weight } & & & $-0.636 * *$ & $-0.576 * *$ \\
\hline Thrips population & & & & $-0.724 * *$ & $-0.670 * *$ \\
\hline Disease incidence & \multirow{2}{*}{$\begin{array}{l}\text { Total dry } \\
\text { matter }\end{array}$} & $-0.203^{\mathrm{NS}}$ & $-0.318^{\mathrm{NS}}$ & $-0.578 * *$ & $-0.592 * *$ \\
\hline Thrips population & & $-0.141^{\mathrm{NS}}$ & $-0.163^{\mathrm{NS}}$ & $-0.582 * *$ & $-0.608 * *$ \\
\hline
\end{tabular}

The mean TDM values of resistant genotypes are $0.32,3.31,11.38$ and 12.45 g plant $^{-1}$ at 20, 40, 60 DAS and at maturity respectively. The mean TDM values of moderately resistant genotypes are $0.28,2.86,7.96$ and $9.31 \mathrm{~g} \mathrm{plant}^{-1}$ at 20,40,60 DAS and at maturity respectively.

The mean TDM values of moderately susceptible genotypes are $0.33,2.92,8.73$ and 9.94 g plant $^{-1}$ at 20, 40, 60 DAS and at maturity respectively. The mean TDM values of susceptible genotypes are $0.26,2.71,8.81$ and $9.71 \mathrm{~g} \mathrm{plant}^{-1}$ at 20, 40,60 DAS and maturity respectively.

Higher TDM values were recorded in the genotypes TU 40 (15.49 $\mathrm{g} \mathrm{plant}^{-1}$ ) followed by LBG 884 (14.11 g plant $^{-1}$ ) and TBG 129 (12.79 g plant $\left.^{-1}\right)$ at maturity which are grouped under resistant category. Lower TDM values at maturity were recorded in the genotypes LBG 951 and LBG 881 (9.08 g plant $^{-1}$ ) preceded by LBG 623 (9.20 g plant $^{-1}$ ) under the moderately resistant category.

Correlation studies revealed that a highly significant negative correlation was observed between the total dry matter and the leaf curl disease incidence at $60 \mathrm{DAS}$ and maturity and a non-significant correlation was observed at 20 DAS and 40 DAS. A highly significant negative correlation was observed between thrips population and total dry matter at 60 DAS and maturity and a non-significant association was observed at 20 DAS and maturity (Table 5).
Dry mass was the only morphological trait significantly correlated with thrips damage in tomato (González, 1974)

Total dry matter accumulation was significantly higher in resistant genotypes indicating that the resistant genotypes were less affected by leaf curl disease caused by GBNV. The susceptible genotypes showed a significant reduction in the total dry matter of the plants. A decrease in the total dry biomass of the plants was observed in that tomato plants infected with yellow mosaic virus (Cladwell, 1934). CMV and BICMV infection has significantly reduced the leaf, stem, pod root ant total dry mass of cowpea varieties (Pio-Ribeiro et al., 1978).

The dry matter accumulation and other four tolerance indicators confirmed antixenosis for the genotype FEB 1115 variety of soybean against thrips (Andrea, 2004). In peanut genotypes the thrips incidence has a lower but negative association with a total dry matter of plant (Ekvised et al., 2006). Soybean genotypes infected with soybean mosaic virus SMV were observed having reduced leaf, stem and root dry weights and severe reduction when infected early (Hema et al., 2015). In groundnut, the plants infected by BICV has reduced the total fresh and dry weights of the plants significantly (Ibrahim et al., 2017). The begomovirus infection in chilli genotype has reduced the shoot, root, stem and total dry matter in susceptible genotypes. 
From the experiment it can be concluded that the leaf curl disease has severely affected the plant growth, development and dry matter partitioning efficiency and screening of genotypes based on the dry matter partitioning and accumulating under naturally epidemic conditions gives the agronomic and physiological performance of those genotypes. The genotype TU 40 showed higher partitioning efficiency.

\section{Acknowledgement}

My first and most earnest, acknowledgment must go to my guide Dr. G. Rama Rao for his Scholastic guidance, unceasing interest, valuable knowledge, technical advice, patient audience. I thank all my teachers and parents for their support and cooperation during my study. I thank my university for providing financial assistance during my research.

\section{References}

ASG.2018. Agricultural statistics at a glance, Government of India, Ministry of Agriculture and Farmers Welfare, Department of Agriculture, Cooperation and Farmers Welfare, Directorate of Economics and Statistics.

Chauhan, J.S., Singh, B.B. and Gupta, S. 2016. Enhancing pulses production in India through improving seed and variety replacement rates. Indian Journal of Genetics and Plant Breeding. 76 (4): 410419.

Biswas, K.K., Tarafdar, A. and Kumar, A.N.U.J. 2009. Multiple infection in urdbean (Vigna mungo) in natural condition by begomovirus, tospovirus and urdbean leaf crinkle virus complex. Indian Phytopathology. 62 (1): 75-82.
Caldwell, J. 1934. The physiology of virus diseases in plants: vi. Some effects of mosaic on the metabolism of the tomato. Annals of Applied Biology. 21 (2): 206224.

Pio-Ribeiro, G., Wyatt, S.D. and Kuhn, C.W. 1978. Cowpea stunt: a disease caused by a synergistic interaction of two viruses. Phytopathology. 68:1260-1265.

Hema, M., Patil, B.L., Chalam, V.C. and Kumar, P.L. 2015. Soybean Mosaic. Virus diseases of tropical and subtropical crops. Virus Diseases of Tropical and Subtropical Crops. 108-120.

Latham, L.J., Jones, R.A.C. and Coutts, B.A., 2004. Yield losses caused by virus infection in four combinations of nonpersistently aphid-transmitted virus and cool-season crop legume. Australian Journal of Experimental Agriculture. 44(1): $57-63$.

Hahn, S.K., Terry, E.R. and Leuschner, K. 1980. Breeding cassava for resistance to cassava mosaic disease. Euphytica. 29 (3): 673683.

González, R.R. 2011. A metabolomics approach to thrips resistance in tomato. Wörhmann Print Service, Zutphen.

Ekvised, S., Jogloy, S., Akkasaeng, C., Keerati K.M., Kesmala, T., Buddhasimma, I. and Patanothai, A. 2006. Heritability and correlation of thrips resistance and agronomic traits in peanut. Asian Journal of Plant Sciences. 5 (6): 923-931.

Ibrahim, A.D., Salaudeen, M.T., Bello, L.Y., Abdullahi, A.A., Adamu, A.S. and Ayeleke, D.A. 2017. Growth and yield components of some groundnut (Arachis hypogaea L.) cultivars infected with blackeye cowpea mosaic virus. Agrosearch. 17 (1): 11-25.

\section{How to cite this article:}

Dinesh Rahul, V., G. Rama Rao, K. Jayalalitha, M. Adinarayana and Srinivasa Rao, V. 2020. Growth and Dry Matter Partitioning Efficiency of Blackgram Genotypes Influenced by Leaf Curl Disease under Natural Field Conditions. Int.J.Curr.Microbiol.App.Sci. 9(09): 1356-1366. doi: https://doi.org/10.20546/ijcmas.2020.909.172 